\title{
Sumoylation of MDC1 is important for proper DNA damage response
}

\author{
Kuntian Luo ${ }^{1,2}$, Haoxing Zhang ${ }^{2}$, \\ Liewei Wang ${ }^{2}$, Jian Yuan ${ }^{1,2, *}$ and \\ Zhenkun Lou ${ }^{2, *}$
}

${ }^{1}$ Key Laboratory of Arrhythmia, Ministry of Education, East Hospital, Tongji University School of Medicine, Shanghai, China and ${ }^{2}$ Division of Oncology Research and Department of Molecular Pharmacology and Experimental Therapeutics, Mayo Clinic, Rochester, MN, USA

In response to DNA damage, many DNA damage factors, such as MDC1 and 53BP1, redistribute to sites of DNA damage. The mechanism governing the turnover of these factors at DNA damage sites, however, remains enigmatic. Here, we show that MDC1 is sumoylated following DNA damage, and the sumoylation of MDC1 at Lys1840 is required for MDC1 degradation and removal of MDC1 and 53BP1 from sites of DNA damage. Sumoylated MDC1 is recognized and ubiquitinated by the SUMO-targeted E3 ubiquitin ligase RNF4. Mutation of the MDC1 Lys 1840 (K1840R) results in impaired CtIP, replication protein A, and Rad51 accumulation at sites of DNA damage and defective homologous recombination (HR). The HR defect caused by MDC1K1840R mutation could be rescued by 53BP1 downregulation. These results reveal the intricate dynamics governing the assembly and disassembly of DNA damage factors at sites of DNA damage for prompt response to DNA damage.

The EMBO Journal (2012) 31, 3008-3019. doi:10.1038/

emboj.2012.158; Published online 25 May 2012

Subject Categories: proteins; genome stability \& dynamics Keywords: homologous recombination; MDC1; RNF4; sumoylation; ubiquitination

\section{Introduction}

DNA damage triggers a series of signal transduction events in cells, collectively called the DNA damage response (DDR) (Zhou and Elledge, 2000). This involves recruitment and activation of proteins that participate in DNA damage sensing, checkpoint signaling, chromatin remodeling, and DNA repair. The recruitment of DNA damage factors to sites of DNA damage is a complex and elaborate process, with different DNA damage factors recruited through distinct processes (Bekker-Jensen et al, 2010; Sy et al, 2010). In response to DNA double-strand breaks (DSBs), the Mre11/

\footnotetext{
*Corresponding authors. J Yuan, Key Laboratory of Arrhythmia, Ministry of Education, East Hospital, Tongji University School of Medicine, Shanghai 200120, China. Tel.: + 862138804518 8344; Fax: + 8621 33901279; E-mail: Yuanjian229@ @otmail.com or Z Lou, Division of Oncology Research and Department of Molecular Pharmacology and Experimental Therapeutics, Mayo Clinic, 200 First Street S W, Rochester, MN 55902, USA. Tel.: + 1507284 2702; Fax: + 1507284 3906; E-mail: lou.zhenkun@mayo.edu
}

Received: 27 March 2012; accepted: 11 May 2012; published online: 25 May 2012
Rad50/NBS1 (MRN) complex detects DSBs and contributes to the recruitment and activation of the PI3K-like kinase (PIKK) ATM (ataxia telangiectasia mutated) (Uziel et al, 2003; Falck et al, 2005; Lee and Paull, 2005). Activated PIKKs phosphorylate histone variant $\mathrm{H} 2 \mathrm{AX}$, which recruits MDC1 to sites of DNA damage (Rogakou et al, 1999; Stucki et al, 2005; Lou et al, 2006). MDC1 in turn acts as an adaptor protein that recruits DDR proteins to sites of damage, such as BRCA1, 53BP1, and the MRN complex (Goldberg et al, 2003; Lou et al, 2003; Stewart et al, 2003). Despite these recent advances, the mechanisms regulating the turnover of DNA damage factors at sites of DNA damage remain largely unknown. It has been reported that MDC1 is ubiquitinated and degraded following DNA damage (Shi et al, 2008). However, the functional significance of MDC1 removal remains unclear. Although Shi et al proposed that removal of MDC1 is a key step for BRCA1 assembly, their conclusion was drawn from experiments using proteasome inhibitor MG132, which may affect many processes during the DDR.

The accumulation of DNA damage factors facilitates DNA repair. There are two major repair pathways that repair DSBs: non-homologous end joining (NHEJ) and homologous recombination (HR) (Warmerdam and Kanaar, 2010). In NHEJ, the two broken ends of DNA are directly ligated without dependence on a homologous template, resulting in quick, but error-prone, repair (Lieber et al, 2004). NHEJ requires the DNA-end-binding $\mathrm{Ku}$ complex, protein kinase DNA-PKcs, potential DNA-end-processing enzymes, such as Artemis, and the XRCC4-Ligase IV complex (Lieber et al, 2003). In contrast to NHEJ, HR is more accurate as the information is copied from an intact homologous DNA sequence; however, as $\mathrm{HR}$ requires an intact sister chromatid, it mainly operates in the S/G2-phases of the cell cycle in mammalian cells (Thompson and Schild, 2002). HR is proposed to occur in several steps. First, the MRN complex regulates the initial resection of the DNA ends with CtIP to produce short 3'-overhangs (Paull and Gellert, 1998; Sartori et al, 2007; Takeda et al, 2007), followed by further resection by Exo1 and Dna2 nucleases to extend the $3^{\prime}$-overhangs (Zhu et al, 2008; Mimitou and Symington, 2009; Nimonkar et al, 2011). The replication protein A (RPA) recognizes and binds the $3^{\prime}$-overhangs, and is then replaced by Rad51 (radiation sensitive 51) with the assistance of other factors (San Filippo et al, 2008). The Rad51-bound single-stranded DNA then moves into the homologous double-stranded DNA (dsDNA) template (strand invasion) (West, 2003). Extension of the invading 3 -strand leads to the formation of Holliday junctions, which are subsequently resolved, to complete an error-free repair of the DSBs (Constantinou et al, 2002; Ip et al, 2008; West, 2009). How the NHEJ and HR pathways coordinate the repair of DSBs remains unclear. However, recent findings that the NHEJ factor 53BP1 blocks DSB resection and the recruitment of Rad51 in BRCA1-deficient cells suggest an antagonistic effect of NHEJ on HR (Bouwman et al, 2010; Bunting et al, 2010). 
The assembly of multiprotein complexes at DNA breaks is tightly controlled. Post-translational protein modifications promote the recruitment and regulate the residence times of most DNA damage factors at damage sites. These modifications include phosphorylation, ubiquitylation, methylation, acetylation, and PARylation (Polo and Jackson, 2011). An emerging theme in DDR is the coordinated roles of sumoylation and ubiquitylation. For example, the SUMO E3 ligases PIAS1 and PIAS4 are required for effective ubiquitin-adduct formation mediated by RNF8 and RNF168 at sites of DNA damage (Galanty et al, 2009; Morris et al, 2009). There are also two Schizosaccharomyces pombe proteins, Rfp1 and Rfp2, which have an N-terminal SUMO-interacting motif (SIM) and a C-terminal RING-finger domain, and play important roles in cell growth and genome stability (Kosoy et al, 2007; Sun et al, 2007). Rfp1 and Rfp2 both lack E3 ubiquitylation activity, but they can recruit Slx8 through a RING-RING interaction. Slx8 is an active RING-finger ubiquitin ligase; thus, together they form a functional E3. In rfp1rfp2 double-null cells, sumoylated proteins were found to be accumulated, which suggests that Rfp/Slx8 proteins may promote ubiquitin-dependent degradation of sumoylated targets (Kosoy et al, 2007; Sun et al, 2007). Similarly, it was reported that in Saccharomyces cerevisiae, Slx5Slx8 is important for preventing the accumulation of DNA damage during normal cell cycle progression (Zhang et al, 2006) and preventing DNA lesions that undergo recombination (Burgess et al, 2007). In addition, the complex inhibits Rad51-independent recombination by modulating the sumoylation of DNA repair proteins (Burgess et al, 2007). RNF4 is the mammalian ortholog of Rfp1/Rfp2. Unlike Rfp1/Rfp2, RNF4 is an active ubiquitin E3 ligase. Both the Rfp-Slx8 complex and RNF4 can ubiquitylate artificial SUMO-containing substrates in a SUMO binding-dependent manner in vitro (Sun et al, 2007). However, the function of RNF4 and its substrates in mammals are not well-characterized. In this study, we found that RNF4 regulates the degradation of sumoylated MDC1, which in turn affects the dissociation of downstream factors from the damage sites as well as the recruitment of other factors. The defect in MDC1 sumoylation results in ineffective DNA repair and prolonged DDR foci. This finding sheds some light on the mechanism regulating the assembly and disassembly of DNA damage factors at sites of DNA damage and the importance of a prompt DDR.

\section{Results}

\section{The sumoylation of MDC1 is enhanced following DNA damage}

While studying DNA damage-induced modifications of $\mathrm{MDC1}$, we found that ectopically expressed MDC1 was sumoylated by SUMO1 (Supplementary Figure 1A), and that the sumoylation of MDC1 was further induced by DNA damage (Supplementary Figure 1B). We also examined MDC1 sumoylation following DNA damage using endogenous MDC1 and SUMO1 (Figure 1A). To confirm that we did detect a covalent reaction rather than an interaction with another sumoylated protein, we include RNF8 as a negative control. As shown in Supplementary Figure 1C, we cannot detect RNF8 in the sumoylation assay samples, which confirmed that the interactions with MDC1-associated proteins were abolished under the condition we used. We could also detect MDC1 sumoylation by SUMO1 and the highly related proteins
SUMO2/3 under denaturing conditions (Figure 1B). These results established that MDC1 is sumoylated by SUMO1/2/3 following DNA damage. Furthermore, by depleting various SUMO E3 ligases using specific shRNAs (Figure 1C and D and Supplementary Figure 1D), we found PIAS4 to be a major E3 ligase involved in the sumoylation of MDC1 following DNA damage. Knocking-down PIAS1-3 failed to abolish MDC1 sumoylation, either due to an insufficient depletion of these proteins or they do not play a major role in MDC1 sumoylation.

We next studied how MDC1 sumoylation is regulated. Using the ATM-specific inhibitor KU55933, we were able to inhibit the radiation-induced sumoylation of MDC1 (Figure 1E), implicating the ATM-mediated damage response pathway in the regulation of MDC1 sumoylation. Furthermore, sumoylation of MDC1 was effectively prevented by the deletion of MDC1's BRCT domain (Supplementary Figure 1E), which is required for MDC1's binding to $\gamma \mathrm{H} 2 \mathrm{AX}$ (Stucki et al, 2005; Lou et al, 2006). Deletion of other regions of MDC1 such as the FHA domain, PST region, or TQXF motifs did not demonstrate similar effects (Supplementary Figure 1E). As the MDC1 BRCT domain is required for the recruitment of MDC1 to sites of DNA damage (Stucki et al, 2005), our results imply that the recruitment of $\mathrm{MDC} 1$ to DNA damage sites is required for its sumoylation. To confirm this, we infected cells with lentivirus encoding H2AX shRNA and found that depletion of H2AX also significantly decreased MDC1 sumoylation (Figure 1F), suggesting that the $\mathrm{MDC} 1-\gamma \mathrm{H} 2 \mathrm{AX}$ interaction is required for MDC1 sumoylation. We also examined the sumoylation of chromatin-associated MDC1 and found that MDC1 sumoylation likely occurs at sites of DNA damage (Figure 1G).

\section{MDC1 is sumoylated at $K 1840$}

Using SUMOplot (http://www.abgent.com.cn/doc/sumoplot/), several candidate MDC1 sumoylation sites were identified. We found that mutation of lysine 1840 to arginine (K1840R) inhibited MDC1 sumoylation (Figure $2 \mathrm{~A}$ and $\mathrm{B}$ ). An in vitro sumoylation assay also showed that MDC1 was sumoylated by SUMO1 (Figure 2C) and SUMO2/3 at K1840 (Figure 2D). Only the WT GST-MDC1 (aa1818-2089) but not the K1840R mutant could be sumoylated in vitro. These data suggest that K1840 is the major DNA damage-induced sumoylation site of MDC1.

\section{The sumoylation of MDC1 regulates its stability}

Protein sumoylation regulates protein stability and localization. Consistent with a previous report (Shi et al, 2008), we found that the MDC1 levels gradually decreased following IR (Figure 3A). Treatment with the proteasome inhibitor MG132 abolished this decrease, suggesting that MDC1 is degraded after IR (Figure 3B). Furthermore, MDC1 was modified by K48-linked ubiquitin chain after IR, as mutation at K48 of ubiquitin abolished MDC1 polyubiquitination (Figure 3C). Interestingly, the MDC1K1840R mutation inhibited the ubiquitination and degradation of MDC1 (Figure 3D-F) with the mutant protein displaying a longer half-life compared to WT MDC1 (Figure 3G, left panel). To exclude the possibility that K1840R mutation could affect turnover of MDC1 in undamaged condition, we examined the half-life of WT MDC1 and K1840R MDC1 in the absence of DNA damage. As shown in Figure 3G (right panel), the half-life of WT and K1840R MDC1 showed no detectable difference. These results suggest that K1840 of MDC1 is required for its ubiquitination and degra- 
A

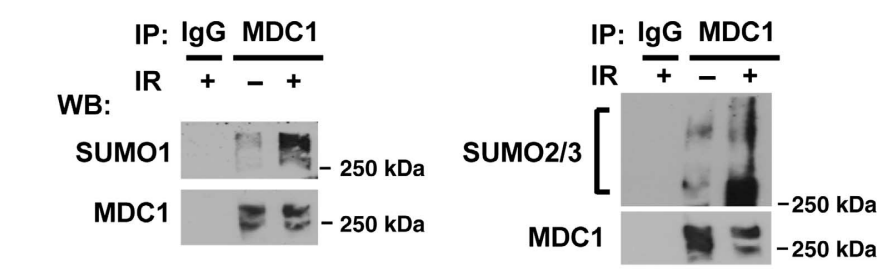

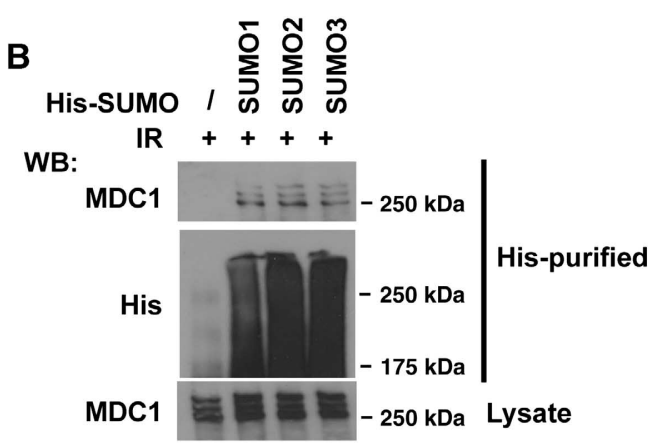

C HA-MDC1 HA-MDC1 + FLAG-SUMO1
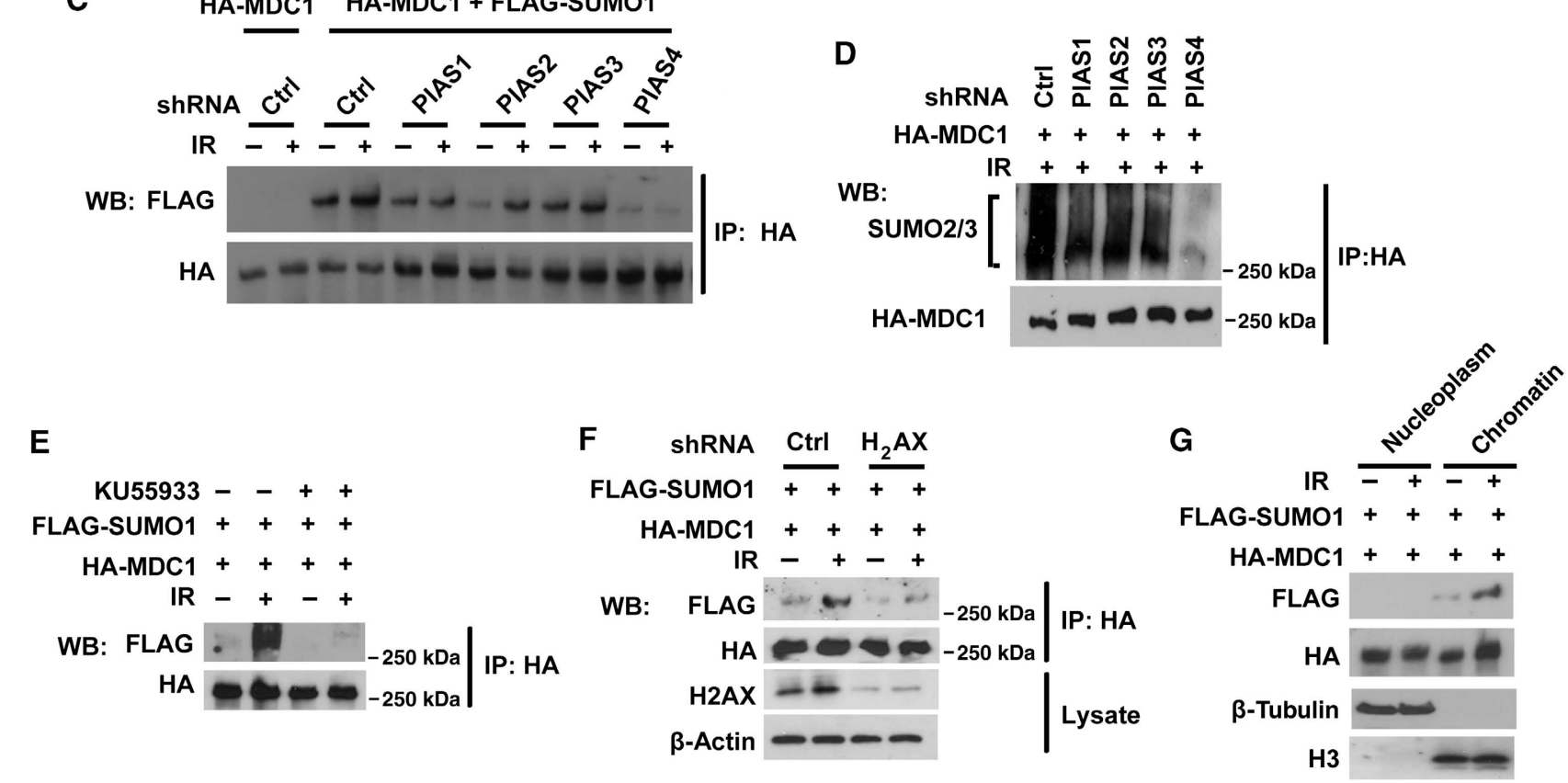

Figure $1 \mathrm{MDC} 1$ is sumoylated following DNA damage. (A) HEK293T cells were left untreated or irradiated (5Gy). MDC1 was then immunoprecipitated with anti-MDC1 antibody, and MDC1 sumoylation was examined as described in Materials and methods using antiSUMO1 or SUMO2/3 antibody. (B) HEK293T cells transfected with His-SUMO1, SUMO2, or SUMO3 were irradiated and harvested $1 \mathrm{~h}$ later. The MDC1 sumoylation was examined under denature conditions. (C, D) MDC1 sumoylation was examined in HEK293T cells depleted of PIAS family E3 ligases. The cells were transfected as indicated and left untreated or irradiated (5 Gy). MDC1 was then immunoprecipitated with antiHA antibody, and MDC1 sumoylation was examined using anti-FLAG antibody (C) or anti-SUMO2/3 antibody (D). (E) DNA damage-induced MDC1 sumoylation was examined in cells pretreated with the ATM inhibitor KU55933 as (C). (F) MDC1 sumoylation was examined in cells infected with H2AX shRNA as in (C). (G) HEK293T cells were transfected and treated as indicated and then subjected to nuclear fractionation. The MDC1 sumoylation was examined in necleoplasma and chromatin-associated fractions

dation following DNA damage. It is possible that K1840 is the major ubiquitination site; however, it is also possible that K1840 of MDC1 is sumoylated, which then targets MDC1 for degradation by ubiquitination. To exclude the possibility that K1840 is a major ubiquitination site, we also generated E1842A mutant, which disrupted the sumoylation consensus sequence in MDC1. As shown in Figure 3H, the E1842A mutation abolished the sumoylation of MDC1 and, importantly, inhibited the ubiqutination of MDC1 (Figure 3I), when K1840 remains intact. Furthermore, when we downregulated PIAS4, which abolished MDC1 sumoylation, MDC1 degradation was also significantly decreased (Figure $3 \mathrm{~J}$ ). The results shown above and next strongly suggest that sumoylation of MDC1 at K1840 is required for MDC1 ubiquitination.

\section{RNF4 targets sumoylated MDC1}

We next investigated which is the E3 required for MDC1 ubiquitination and degradation. Among potential E3 ubiqui- tin ligases, mammalian RNF4 is unique in that it contains a tandem array of four SIMs near its N-terminus making it a SUMO-targeted ubiquitin ligase (STUL) (Prudden et al, 2007; Perry et al, 2008; Tatham et al, 2008a). We thus speculated that RNF4 might be the link between MDC1 sumoylation and ubiquitination. Indeed, we found that MDC1 interacted with endogenous RNF4 and that DNA damage enhanced this interaction (Figure 4A). Furthermore, mutation of MDC1 K1840 or deletion of the SIMs of RNF4 abolished the MDC1-RNF4 interaction (Figure 4B and C). The recruitment of RNF4 to chromatin also requires MDC1K1840 (Supplementary Figure 2A). These results suggest that the RNF4-MDC1 interaction is mediated by the SIMs and sumoylated K1840. To determine whether RNF4 is required for MDC1 degradation following IR, we downregulated RNF4 using RNF4 siRNA. Knockdown of RNF4 significantly decreased the K48-linked polyubiquitination of MDC1 (Figure 4D). We also found that knockdown of RNF4 sig- 

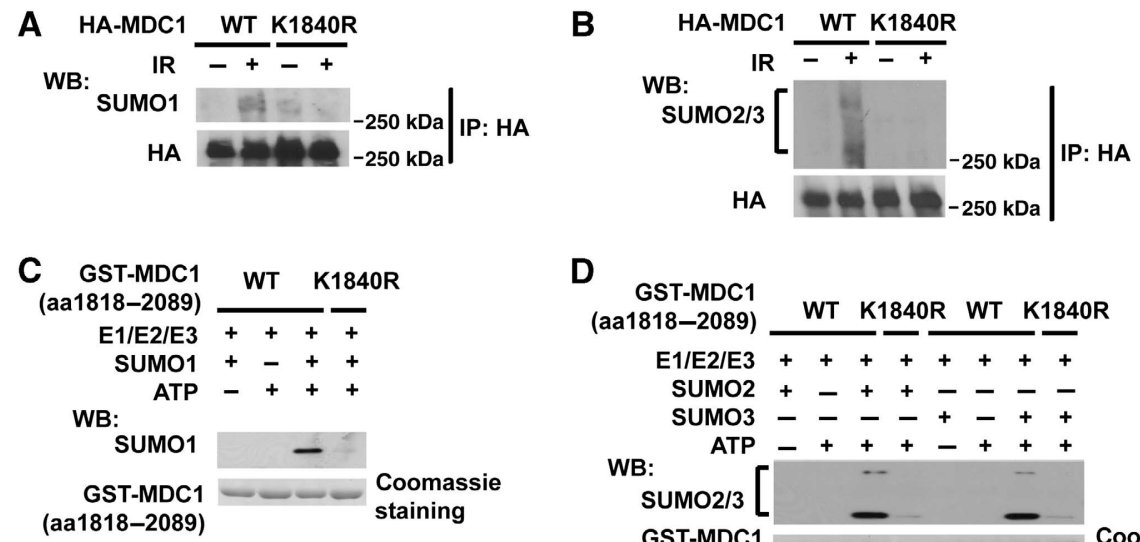

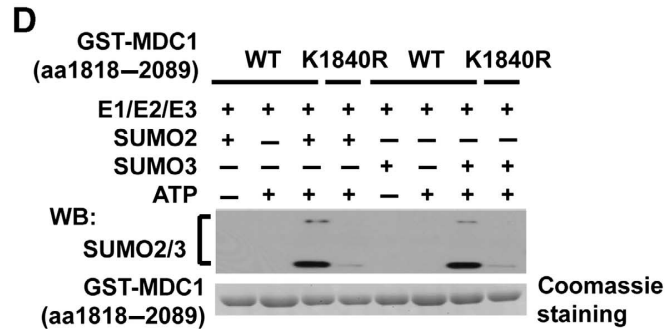

Figure 2 Sumoylation of MDC1 on K1840 in vivo and in vitro. (A, B) HEK293T cells transfected with HA-MDC1-WT or the HA-MDC1-K1840R mutant were treated and subjected to immunoprecipitation and immunoblot as indicated. (C, D) Bacterially expressed GST-MDC1 (aa 1818-2089) WT or K1840R bound to GST-sepharose were subjected to in vitro sumoylation reactions in the presence or absence of SUMO1, SUMO2, SUMO3, or ATP as indicated.

nificantly decreased the MDC1 degradation (Figure 4E) while knockdown of RNF8 showed no effect on MDC1 degradation (Supplementary Figure 2B). Reconstituting RNF4-deficient cells with WT RNF4, but not RNF4 lacking the SIMs motif, restored IR-induced MDC1 degradation (Supplementary Figure 2C).

To further confirm that RNF4 targets sumoylated MDC1, we performed in vitro sumoylation assay followed with in vitro ubiquitination assay (Figure 4F). As shown in Figure 4G and Supplementary Figure 2D, GST-MDC1 (aa1818-2089) could be sumoylated in vitro by SUMO1/2/3 and then ubiquitinated by WT, but not catalytically inactive RNF4 (RNF4-CS). However, unsumoylated GST-MDC1 (aa1818-2089) could not be ubiquitinated (Figure 4G, lane 6 ), suggesting that prior sumoylation of MDC1 is required for subsequent ubiquitination by RNF4. In support of this, deletion of the SIM domain of RNF4 abolished MDC1 ubiquitination (Figure 4G, lane 5). Furthermore, mutation at K1840 of MDC1 abolished its sumoylation and ubiquitination (Figure $4 \mathrm{H}$ ). These results established that the sumoylation of $\mathrm{MDC} 1$ at $\mathrm{K} 1840$ is required for MDC1 ubiquitination by RNF4.

These results strongly suggest that $\mathrm{MDC} 1$ is ubiquitinated by RNF4 in a sumoylation-dependent manner, which subsequently leads to the proteasome-dependent degradation of MDC1.

\section{MDC1 sumoylation is important for $H R$}

We next investigated how a defect in MDC1 sumoylation and degradation would affect DDR. Using a reporter assay for HR (Moynahan et al, 2001), we found that siRNA-mediated downregulation of MDC1 resulted in compromised HR (Figure 5A), consistent with previous reports (Zhang et al, 2005; Xie et al, 2007). Reconstituting cells with siRNAresistant WT MDC1 rescued the HR defect. However, MDC1K1840R could not rescue the HR defect, suggesting that MDC1 sumoylation is important for HR. On the other hand, we did not find a significant effect of MDC1 sumoylation on NHEJ (Figure 5B). In addition, cells expressing MDC1K1840R were more sensitive to DNA damage (Figure 5C).

Downregulation of RNF4 also resulted in a defect in HR (Figure 5D). However, downregulation of RNF4 in cells expressing MDC1 K1840R did not induce a further defect in HR (Figure 5D). Unlike cells expressing MDC1K1840R, cells depleted of RNF4 had a defect in NHEJ (Figure 5E). Cells depleted of RNF4 are also hypersensitive to IR and showed more sensitivity than cells expressing MDC1K1804R (Figure 5F, G). On the other hand, introducing MDC1K1840R mutation in cells depleted of RNF4 did not further sensitize these cells to IR (Figure 5G). These results suggest that RNF4 is important for the DDR. Sumoylated MDC1 is a major target of RNF4 in regulating the HR pathway, and RNF4 may have other targets in regulating the NHEJ pathway during DNA repair.

\section{Defective DSB resection in cells expressing MDC1 sumo mutant}

We further examined how mutation at the MDC1 sumoylation site results in defective HR. Interestingly, when we examined Rad51 recruitment at sites of DNA damage, we found that Rad51 foci formation was defective in MDC1 - / - mouse embryonic fibroblasts (MEFs) stably reconstituted with K1840R mutant compared to that of WT MDC1 (Figure 6A and $\mathrm{B}$ ). The chromatin binding of Rad51 was also defective in cells expressing MDC1K1840R (Supplementary Figure 3A). We further investigated upstream events leading to Rad51 recruitment, and found that the accumulation of CtIP and RPA at sites of DNA damage is defective in cells expressing MDC1K1840R (Figure 6C and D and Supplementary Figure 3B, C). Since CtIP is required for DNA end resection (Sartori et al, 2007), our results suggest that MDC1 sumoylation has a pivotal role in the regulation of DSB resection and HR.

\section{The sumoylation of MDC1 affects HR through 53BP1}

We next asked how MDC1 sumoylation regulates the recruitment of CtIP, RPA, and Rad51. The initial characterization of the K1840R mutant showed that sumoylation of MDC1 did not affect MDC1's ability to relocalize to sites of DNA damage (Figure 7A). In a time course study of MDC1 foci formation and nuclear fractionation following DNA damage, we found that the MDC1 K1840R mutant showed sustained accumulation at sites of DNA damage compared to WT MDC1 (Figure 7A and B, Supplementary Figure 3D), suggesting 
that MDC1 sumoylation at K1840 is not important for MDC1 foci formation following DNA damage, but rather the sustained MDC1 accumulation at sites of DNA damage. In cells depleted of RNF4 and PIAS4, MDC1 foci are also sustained (Supplementary Figures 3E, F). These results are consistent with sustained MDC1 level in these cells. We also found that the dynamics of 53BP1 foci formation was similar to that of MDC1 in MEFs and U2OS cells (Figure 7A and B and
Supplementary Figure 4A). The BRCA1 foci were also slightly sustained in cells expressing MDC1K1840R mutant (Figure 7C). Recent findings suggest that H2AX/MDC1/ 53BP1 is antagonistic for HR (Bouwman et al, 2010; Bunting et al, 2010; Helmink et al, 2011). Consistent with this, the appearance of Rad51 foci coincided with the disappearance of MDC1 and 53BP1 foci (Figure 6B versus Figure 7B). Even in cells with both 53BP1 and Rad51 foci,
A

IR: $\quad \begin{array}{llllll}0 & 1 & 4 & 8 & 12 & \text { (h) }\end{array}$

WB:

MDC1

B-Actin

B

D

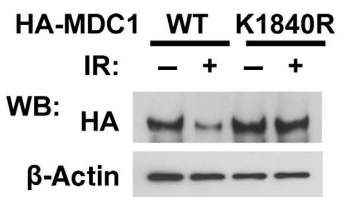

E

HA-MDC1 WT K1840R

IR -+-+

WB:

anti-Ub

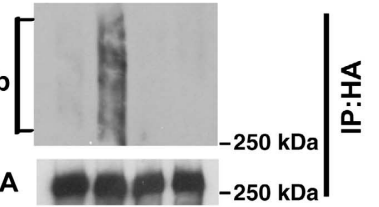

c

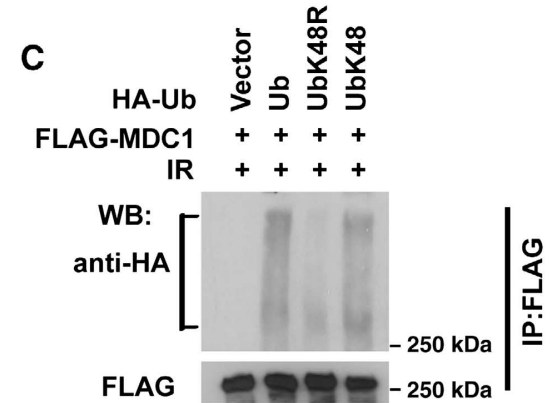

$\mathbf{F}$

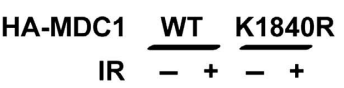

WB:

Ub-K48

R + +

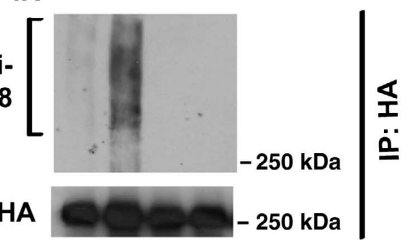

G
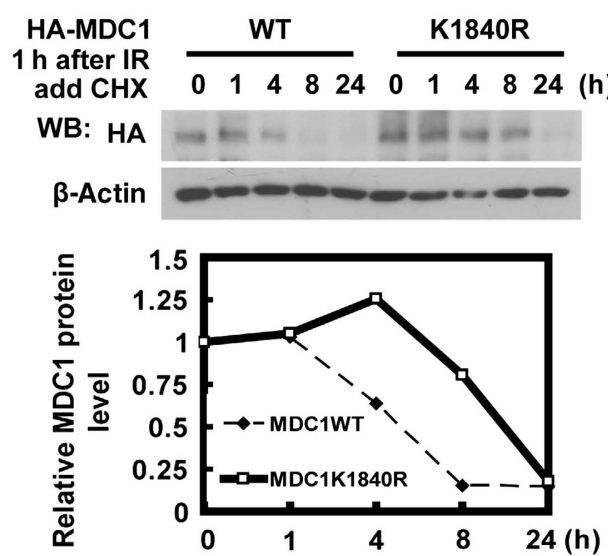

H

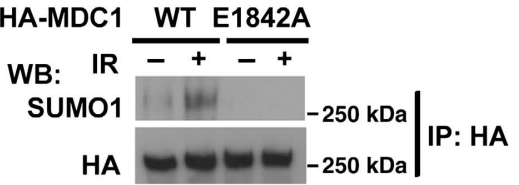

I HA-MDC1 WT E1842A

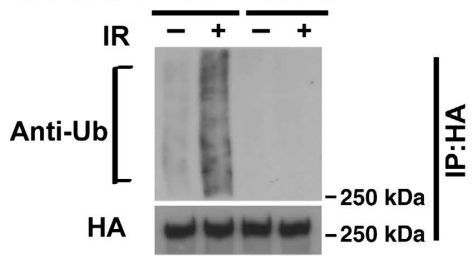

(h)
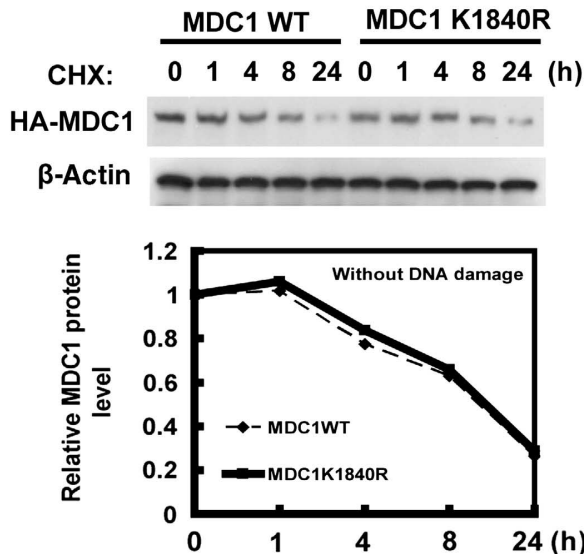

J

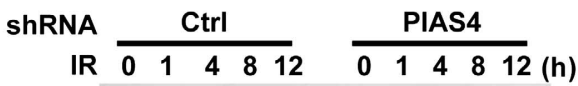

WB:

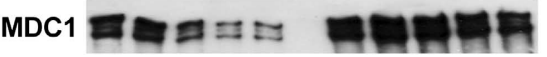

PIAS4 na ba net

$\beta$-Actin

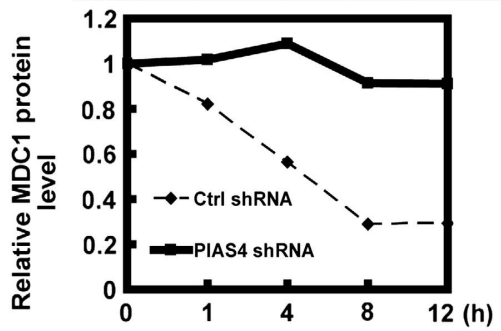


they tend not to colocalize (Supplementary Figure 4A). Therefore, it is possible that the sustained MDC1/53BP1 accumulation is detrimental for CtIP/RPA/Rad51 recruitment. To test this hypothesis, we downregulated 53BP1 in cells expressing WT MDC1 or K1840R. As shown in Figure 7D and E, downregulation of 53BP1 (Supplementary Figure 4B) in cells expressing WT MDC1 did not have a significant effect on Rad51 foci formation, except an enhanced Rad51 foci formation at an early time point. Dramatically, downregulation of 53BP1 in cells expressing MDC1K1840R rescued the defects in Rad51 foci formation. Importantly, downregulation of 53BP1 in cells expressing MDC1K1840R could rescue the HR defect (Figure 7F). There was no statistical difference in HR between cells expressing WT MDC1 and MDC1K1840R when 53BP1 was depleted (column 3 and 4 Figure 7F; $P=0.309$ ). Similarly, there was no statistically significant difference in Rad51 recruitment between these two groups (Figure 7E; $P>0.25$ ). These data strongly suggest that MDC1 sumoylation regulates the HR pathway mainly through 53BP1.

In summary, our findings suggest that sumoylation and ubiquitination of MDC1 operate in a sequential manner to regulate DNA repair. Upon DNA damage, MDC1 is recruited to chromatin to facilitate the DDR. MDC1 is then sumoylated by PIAS4, and sumoylated MDC1 is targeted by RNF4, resulting in the ubiquitination and degradation of MDC1. The sumoylation of MDC1 is important for the disassembly of MDC1 and 53BP1 from sites of DNA damage, DSB resection, and HR. These results reveal important insights into the complex dynamics of assembly and disassembly of DNA damage factors at sites of DNA damage, facilitating a prompt DDR.

\section{Discussion}

In eukaryotic cells, DSBs activate the DDR, which is a complex signaling network that regulates cell cycle progression, transcription, DNA repair, and cell survival (Harper and Elledge, 2007). A number of factors involved in the DDR are recruited to DSBs to promote DNA repair. MDC1 is an important nodule that helps recruit DDR factors (Stucki and Jackson, 2004; Jungmichel and Stucki, 2010). How MDC1 itself is recruited to the DNA damage sites has been well studied. However, the mechanism by which MDC1 disassociates from the foci is not clear. It has been reported that MDC1 is ubiquitinated and degraded following DNA damage (Shi et al, 2008), although the mechanism responsible for MDC1 ubiquitination is unclear. Our results suggest that an active removal of MDC1 from chromatin by
RNF4 by sumoylation-coupled ubiquitination is a major mechanism for MDC1 removal. The reasons for this conclusion include the following: first, MDC1 sumoylation is targeted for its degradation by RNF4 (Figure 3 and 4); second, MDC1 sumoylation requires its localization to sites of DNA damage (Figure 1F and Supplementary Figure 1E); and third, RNF4 recruitment to the chromatin requires MDC1 sumoylation at K1840 (Supplementary Figure 2A). We think that a positive-feedback loop is responsible for sustained MDC1 foci in cells expressing the MDC1 sumo mutant. On the one hand, the MDC1 sumo mutant, which cannot be degraded by RNF4, could not be removed by RNF4 from chromatin. On the other hand, mutation at the MDC1 sumoylation site causes HR defect, which in turn induces more sustained MDC1 foci. The findings that MDC1 could only be sumoylated and degraded at sites of DNA damage also explains modest and delayed MDC1 degradation following DNA damage, since MDC1 in the nucleoplasm is not subjected to sumoylation and RNF4-mediated degradation. Although decreased MDC1 levels are not rapidly detected by western blot, one could envision a dynamic process of MDC1 degradation and recruitment at DSBs, and that MDC1 degradation occurs much earlier following DNA damage.

We found that cells expressing MDC1K1840R show defects in CtIP/RPA/Rad51 recruitment to sites of DNA damage. One potential mechanism is that MDC1 sumoylation at K1840 is directly involved in the recruitment of CtIP/RPA/Rad51. Several lines of evidence argue against this possibility. First, sumoylation at MDC1K1840 results in RNF4 binding and MDC1 degradation. Second, HR defects caused by MDC1K1840R mutation could be rescued by 53BP1 depletion, suggesting that sustained $53 \mathrm{BP} 1$ on chromatin contributes to the HR defects. A previous study by Shi et al suggests that removal of $\mathrm{MDC} 1$ is required for BRCA1 recruitment, while we found that BRCA1 recruitment is normal in cells with sustained MDC1. Shi et al used MG132 to sustain MDC1 foci and performed all the functional assays, which may affect lots of processes during DDR. Our studies are consistent with recent ground-breaking studies suggesting 53BP1 has an antagonistic role against HR (Bouwman et al, 2010; Bunting et al, 2010). A recent study also suggests that $\mathrm{H} 2 \mathrm{AX}$ and $\mathrm{MDC} 1$ could prevent DNA end resection by CtIP in G1-phase cells (Helmink et al, 2011). However, a mechanism to relieve this inhibition of DSB resection for post-replicative cells should be available, as HR is the preferred pathway for post-replicative cells. Our studies provide a potential mechanism to relieve the inhibition of DSB resection by the H2AX-MDC1-53BP1

\footnotetext{
Figure 3 The sumoylation of MDC1 regulates its stability following DNA damage. (A) MDC1 levels were examined at indicated time points in cells treated with IR $(5 \mathrm{~Gy})$. (B) MG132 treatment reversed the degradation of MDC1 after IR. U2OS cells were treated with MG132 $\left(20 \mu \mathrm{g} \mathrm{ml}^{-1}\right)$, and left unirradiated or irradiated (5 Gy). Whole-cell lysates were prepared $8 \mathrm{~h}$ following the treatment. (C) HEK293T cells transfected with indicated constructs were pretreated with MG132 $\left(20 \mu \mathrm{g} \mathrm{ml}^{-1}\right)$ followed by IR (5 Gy). After $3 \mathrm{~h}$, cells were subjected to immunoprecipitation and immunoblot as indicated. (D) $\mathrm{MDC}^{-/-}$MEFs reconstituted with WT or K1840R HA-MDC1 were left untreated or treated with IR (5 Gy), and MDC1 levels were examined $8 \mathrm{~h}$ later. (E, F) MDC1 ${ }^{-/}$- MEFs reconstituted with WT or K1840R HA-MDC1 were treated with MG132 $\left(20 \mu \mathrm{g} \mathrm{ml}^{-1}\right)$ followed by irradiation. MDC1 ubiquitination was examined $3 \mathrm{~h}$ later by immunoprecipitation and immunoblots as indicated. (G) K1840R MDC1 has longer half-life compared to WT MDC1. MDC1 ${ }^{-/-}$MEFs reconstituted with WT or K1840R HA-MDC1 were irradiated (5 Gy) (left panel) or unirradiated (right panel) followed with CHX (100 $\mu \mathrm{g} \mathrm{ml}{ }^{-1}$ ) treatment. MDC1 levels were examined at indicated time points. Bottom panels: quantification of MDC1 protein level with Image J. (H) HEK293T cells transfected with HA-MDC1-WT or the HA-MDC1-E1842A mutant were treated and subjected to immunoprecipitation and immunoblot as indicated. (I) HEK293T cells transfected with HA-MDC1-WT or the HA-MDC1-E1842A mutant were treated with MG132 $\left(20 \mu \mathrm{g} \mathrm{ml}^{-1}\right)$ followed by irradiation. MDC1 ubiquitination was examined $3 \mathrm{~h}$ later by immunoprecipitation and immunoblots as indicated. (J) U2OS cells were infected with Ctrl or PIAS4 shRNA. After $48 \mathrm{~h}$, cells were treated with IR ( $5 \mathrm{~Gy}$ ), and MDC1 levels were examined at indicated time points. Bottom panel: quantification of MDC1 protein level with Image J.
} 
A

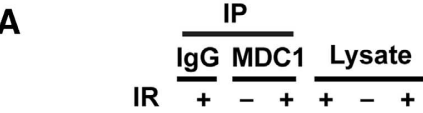

WB: RNF4 MDC1

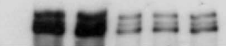

B

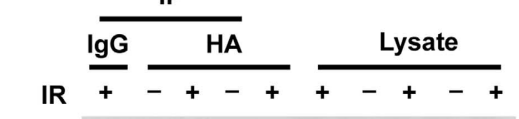

WB: RNF4

$-$

C

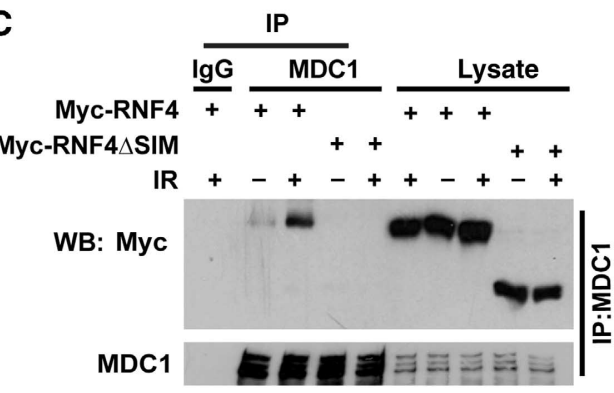
E

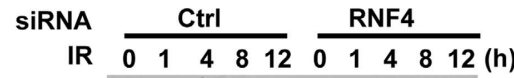

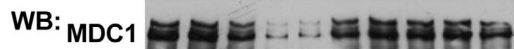
RNF4
$\beta$-Actin

\section{SiRNA Ctrl RNF4 \\ HA-MDC1 ++++ \\ IR -+-+}

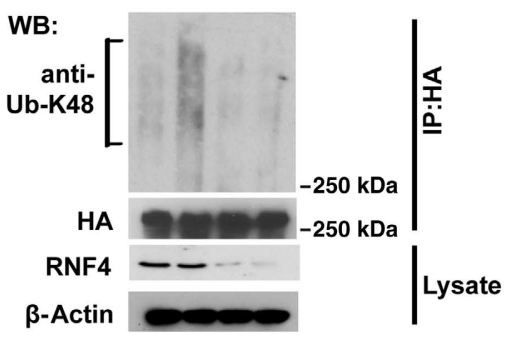

F In vitro sumoylation assay $\rightarrow$ Wash beads $\rightarrow$ In vitro ubiquitination assay $\rightarrow$ Wash beads \begin{tabular}{|l|l|l|l|}
\hline Frist step & & Second step & \\
\hline
\end{tabular}

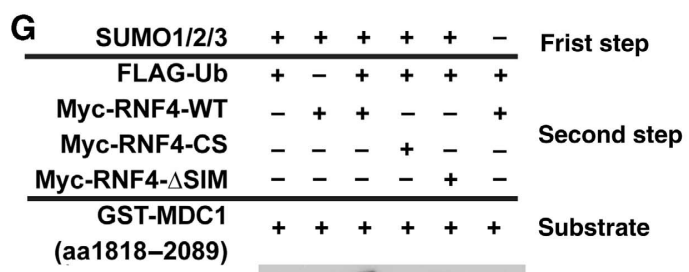

H
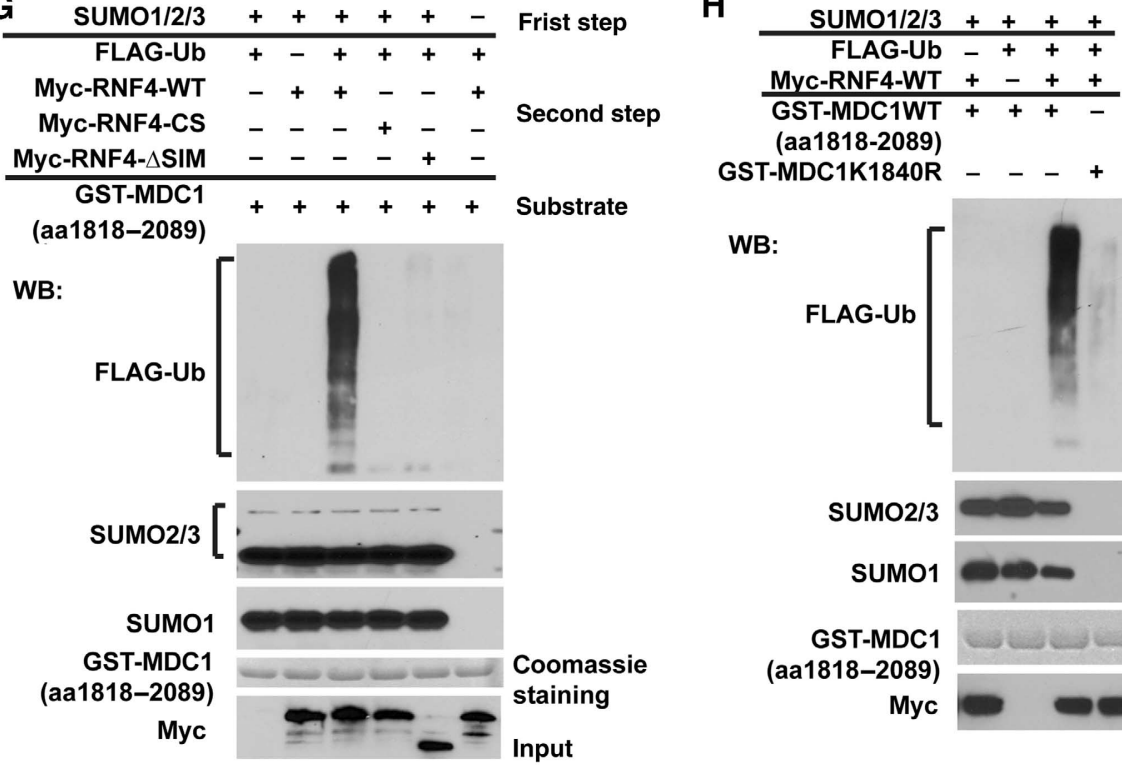

WB:

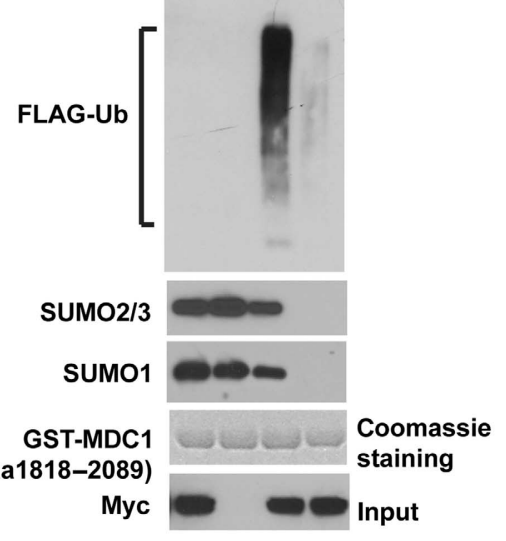

Figure 4 The sumo-dependent degradation of MDC1 following DNA damage is mediated by RNF4 ubiquitination. (A, B) HEK293T cells (A) or MDC1 $^{-/-}$MEFs reconstituted with HA-WT MDC1 or HA-K1840R (B) were treated with MG132 and left unirradiated or irradiated, and the MDC1-RNF4 interaction was examined $4 \mathrm{~h}$ later by immunoprecipitation and immunoblot as indicated. (C) HEK293T transfected with MycRNF4 or RNF4 $\triangle$ SIM mutant were treated as (A) and subjected to immunoprecipitation and immunoblot as indicated. (D) MDC1 ubiquitination was examined in HEK293T cells transfected with control or RNF4 siRNA as Figure 3F. (E) U2OS cells transfected with Ctrl or RNF4 siRNA were treated with IR (5 Gy), and MDC1 levels were examined at indicated time points. (F) Schematic of the combined in vitro sumoylation and ubiquitination assay procedures. (G, H) Bacterially expressed GST-MDC1(aa 1818-2089) WT or K1840R bound to GST-sepharose were subjected to combined in vitro sumoylation and ubiquitination reactions as shown in (F). The GST-MDC1(aa 1818-2089) WT (G) was subjected to in vitro sumoylation reactions in the presence or absence of SUMO1, SUMO2, and SUMO3, and then subjected to in vitro ubiquitination reactions in the presence or absence of FLAG-Ub, RNF4-WT, RNF4-CS, or RNF4 $\Delta$ SIM mutant as indicated. GST-MDC1(aa 1818-2089) WT or K1840R (H) was subjected to in vitro sumoylation reactions in the presence of SUMO1, SUMO2, and SUMO3, and then subjected to in vitro ubiquitination reactions in the presence or absence of RNF4 or FLAG-Ub as indicated. The ubiquitination and sumoylation of GST-sepharosebound protein were analyzed by immunoblot as indicated. 
A
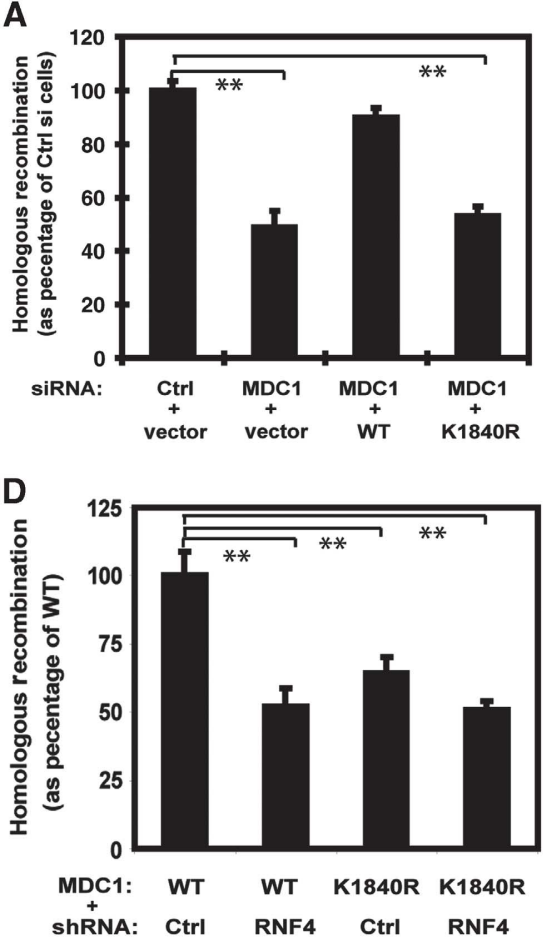

B

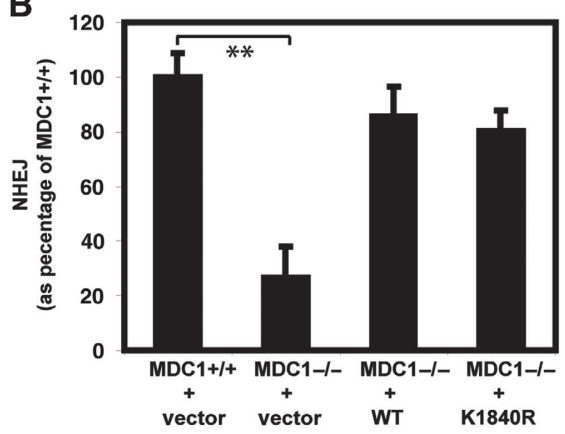

E

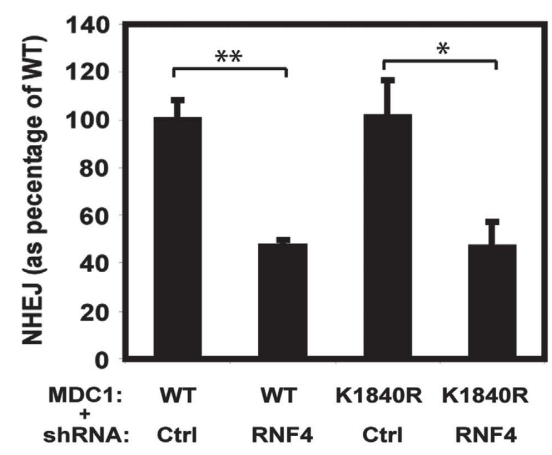

C

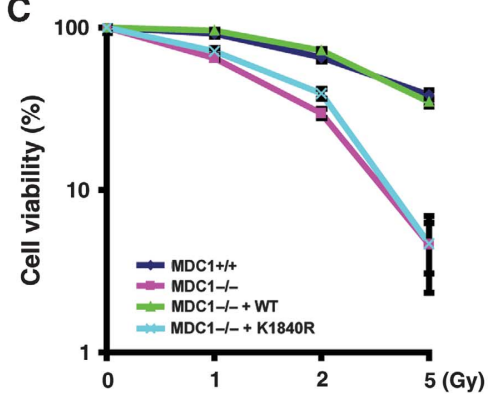

$\mathbf{F}$

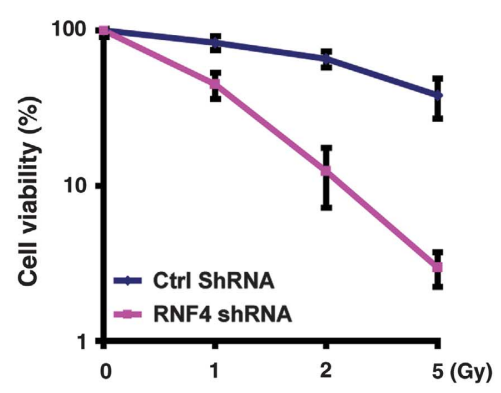

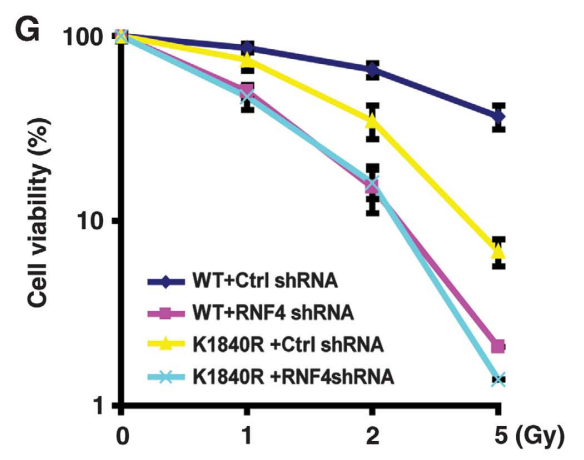

Figure 5 Sumoylation of MDC1 on K1840 is important for the HR DNA repair and DDR. (A, B) Effects of MDC1 sumoylation on HR (A) and NHEJ (B); Error bars represent s.e.m. from three independent experiments. ${ }^{*} P<0.01$ two-tailed Student's $t$-test. (C) $\mathrm{MDC1}^{+/+}$, MDC1 ${ }^{-/-}$, and $\mathrm{MDC1}^{-/-}$MEFs reconstituted with WT or K1840R MDC1 were irradiated and colony formation assay was performed. Error bars represent s.e.m. from three independent experiments. (D, E) MDC1 ${ }^{-1}$ MEFs reconstituted with WT or K1840R FLAG-MDC1 were infected with Ctrl or RNF4 shRNA. The cells were subjected to HR (D) or NHEJ (E) assay as described in method. Error bars represent s.e.m. from three independent experiments. ${ }^{* *} P<0.01,{ }^{*} P<0.05$ two-tailed Student's $t$-test. (F) Control cells and cells depleted RNF4 with shRNA were irradiated and colony formation assay was performed. Error bars represent s.e.m. from three independent experiments. (G) MDC1 ${ }^{-/-}$MEFs reconstituted with WT or K1840R MDC1 were stably depleted RNF4 with shRNA or control shRNA. Cells were irradiated and colony formation assay was performed. Error bars represent s.e.m. from three independent experiments.

pathway. Future studies are required to determine how RNF4 activity is regulated in a DNA damage- and cell cycledependent manner.

Previous studies have mainly focused on how sumoylation is required for the recruitment of DDR factors to the damage sites, such as the human BRCA1, BLM, XRCC4, and RPA, and budding yeast Sgs1, Ku70, and Rad52 (Eladad et al, 2005; Yurchenko et al, 2006; Galanty et al, 2009; Morris et al, 2009; Dou et al, 2010; Polo and Jackson, 2011). Our study showed that sumoylation also regulates the disassociation of DDR factors by working together with ubiquitination during DNA damage in a coordinated manner. This is achieved by a unique SUMO-targeted E3 ubiquitin ligase RNF4. The homolog of RNF4 in yeast has been implicated in DDR and maintenance of genomic stability (Sun et al, 2007), and mammalian RNF4 has been shown to regulate PML levels (Lallemand-Breitenbach et al, 2008; Tatham et al, 2008b). However, the function of RNF4 in the DDR in mammals is still unclear. Our study showed that RNF4 regulates both NHEJ and HR. RNF4-deficent cells are not able to recover from DNA damage and are hypersensitive to radiation. We further showed that MDC1 is a major target of RNF4 in regulating $\mathrm{HR}$, as depleting RNF4 in cells expressing the MDC1 sumo mutant did not further compromise HR. On the other hand, MDC1 sumoylation did not affect NHEJ, suggesting that RNF4 regulates NHEJ through other factors. This will be another important question to be addressed by future studies.

In summary, our studies revealed the intricate dynamics of the assembly and disassembly of DNA damage factors 
A
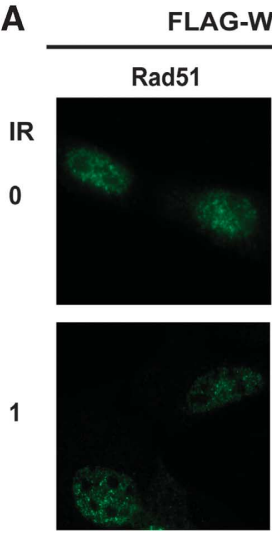

8

(h)

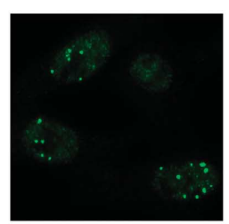

C

IR

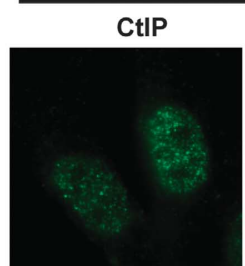

FLAG-MDC1-WT

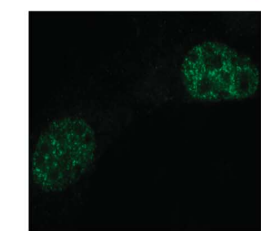

4

(h)

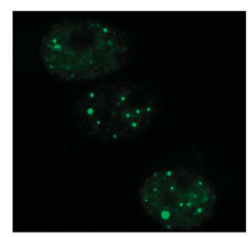

FLAG-K1840R-MDC1
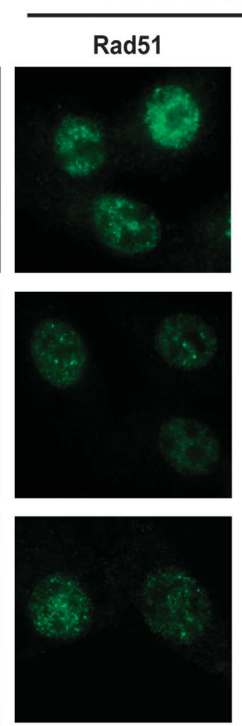

FLAG-MDC1-K1840R
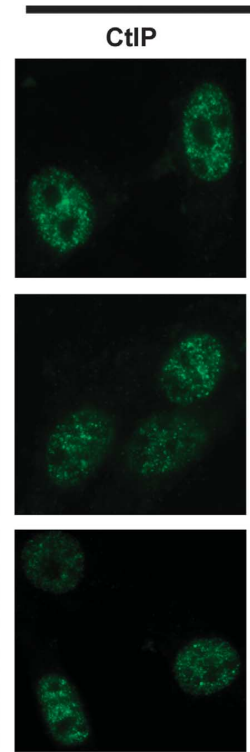

FLAG
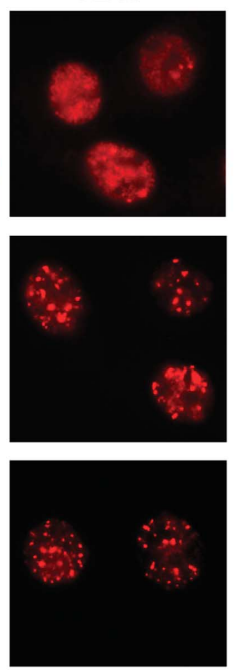

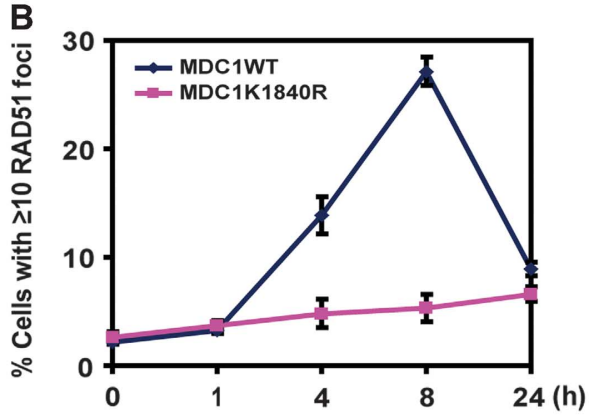

D

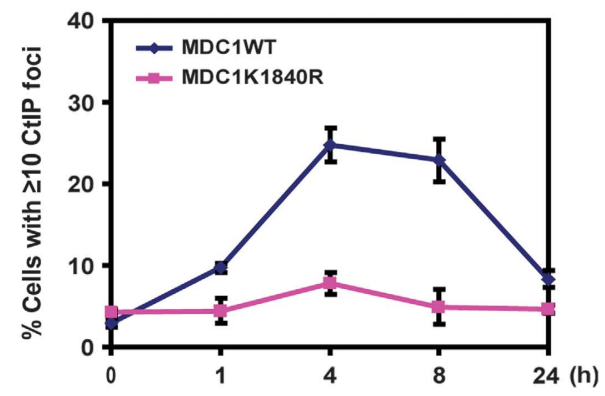

Figure 6 MDC1 sumoylation regulates MDC1 and Rad51 accumulation at DSBs. (A, B) MDC1 ${ }^{-/-}$MEFs reconstituted with WT or K1840R FLAG-MDC1 were left untreated or irradiated ( $2 \mathrm{~Gy}$ ) and probed with indicated antibodies at different time points; representative images are shown in (A); the quantification of the percentage of cells displaying Rad51 foci is shown in (B). Error bars represent s.e.m. from three independent experiments. (C, D) $\mathrm{MDC1}^{-/}$MEFs reconstituted with WT or K1840R FLAG-MDC1 were left untreated or irradiated (2 Gy) and probed with indicated antibody at different time points. Representative images are shown in (C), the quantification of the percentage of cells displaying CtIP foci are shown in (D). Error bars represent s.e.m. from three independent experiments.

as well as the close coordination of sumoylation and ubiquitination at sites of DNA damage, which is critical for a prompt DDR.

\section{Materials and methods}

\section{In vivo sumoylation assay}

Transfected HEK293T cells were lysed in $120 \mu \mathrm{l}$ of $62.5 \mathrm{mM}$ Tris-HCl (pH 6.8), $2 \%$ SDS, $10 \%$ glycerol, $20 \mathrm{mM} \mathrm{N}$-ethylmaleimide (NEM), boiled for 15 min, diluted 10 times with NETN buffer containing protease inhibitors and $20 \mathrm{mM} \mathrm{NEM}$, and centrifuged (16000 g, $10 \mathrm{~min}, 4^{\circ} \mathrm{C}$ ) to remove cell debris. With these conditions, the interaction between MDC1 and other possible sumoylated proteins should be disrupted. The lysates were immunoprecipitated with
EZview Red Anti-HA affinity gel (Sigma) or with anti- FLAG M2 affinity gel (Sigma) for $1.5 \mathrm{~h}$ at $4^{\circ} \mathrm{C}$ with rocking, and the precipitates were washed and eluted in SDS sample buffer. Western blots were analyzed with indicated antibodies. A covalent reaction but not an interaction with another sumoylated protein should be detected.

\section{In vivo sumoylation assay under denature conditions}

Transfected HEK293T cells were lysed in $120 \mu \mathrm{l}$ of $62.5 \mathrm{mM}$ Tris- $\mathrm{HCl}$ (pH 6.8), $2 \%$ SDS, $10 \%$ glycerol, $20 \mathrm{mM} \mathrm{NEM}$, boiled for $15 \mathrm{~min}$, diluted 10 times with NETN buffer containing $6 \mathrm{M}$ guanidium- $\mathrm{HCl}$, $10 \mathrm{mM}$ imidazol $\mathrm{pH} 8$, protease inhibitors, and $20 \mathrm{mM}$ NEM, and centrifuged $\left(16000 \mathrm{~g}, 10 \mathrm{~min}, 4^{\circ} \mathrm{C}\right)$ to remove cell debris. The lysates were immunoprecipitated with TALON Cobalt Resin (Clontech) for $1.5 \mathrm{~h}$ at $4{ }^{\circ} \mathrm{C}$ with rocking and the precipitates were washed with 
A

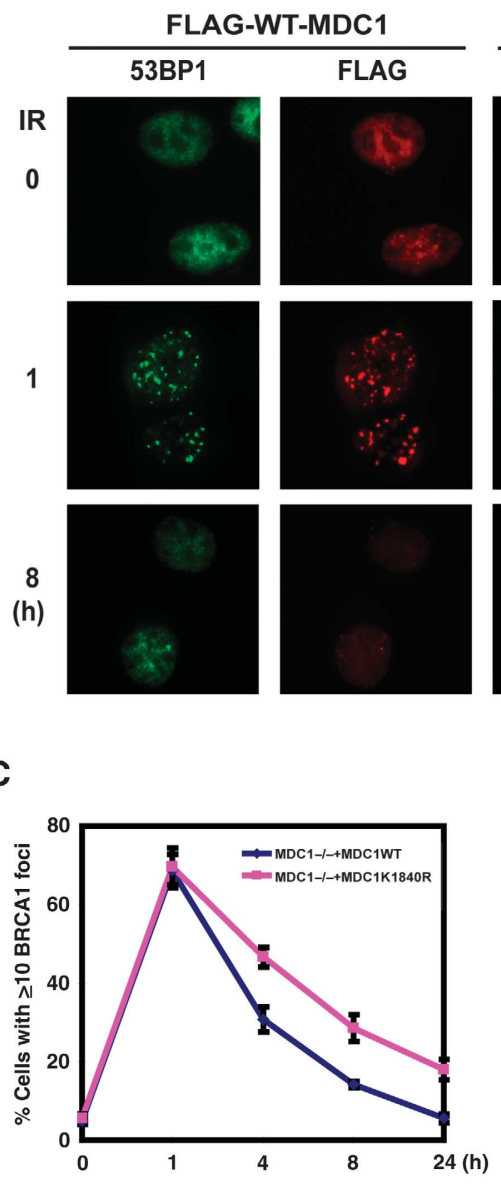

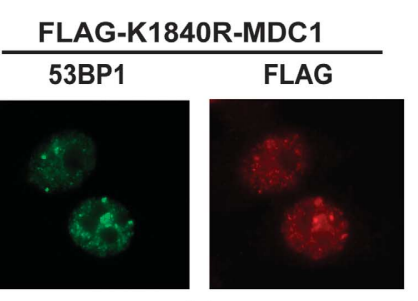
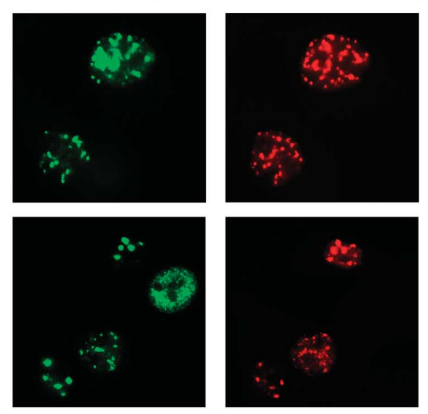

E

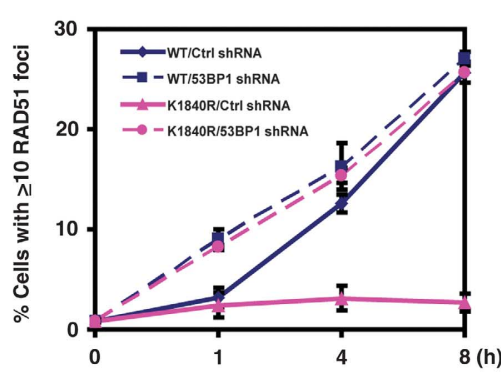

B
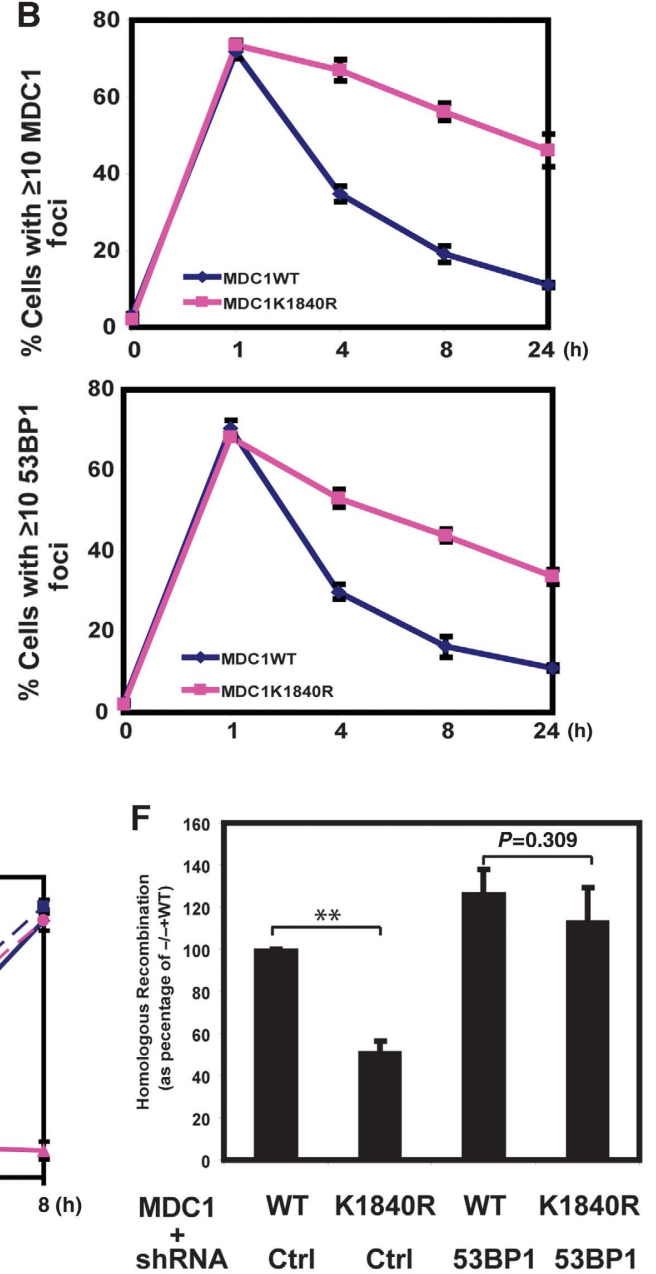

D

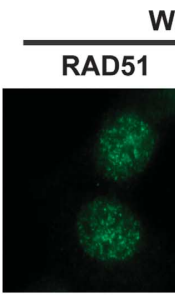

WT/Ctrl ShRNA
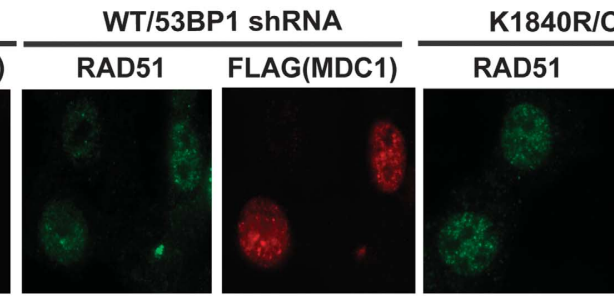

trl shRNA \begin{tabular}{ll} 
K1840R/53BP1 shRNA \\
\hline RAD51 FLAG(MDC1)
\end{tabular}

1
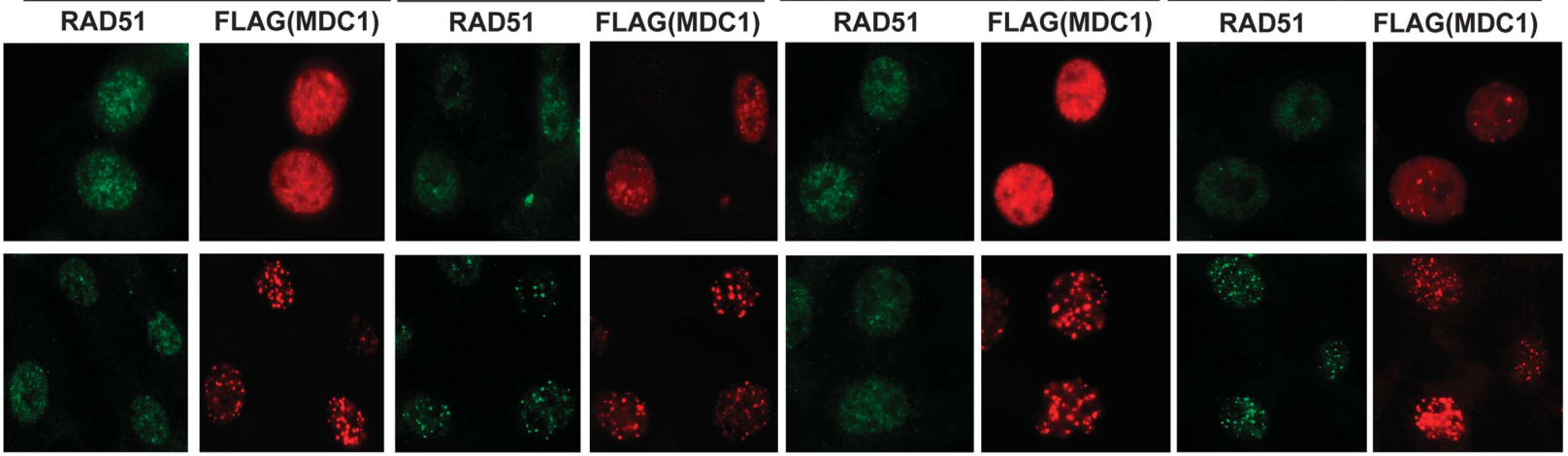

8
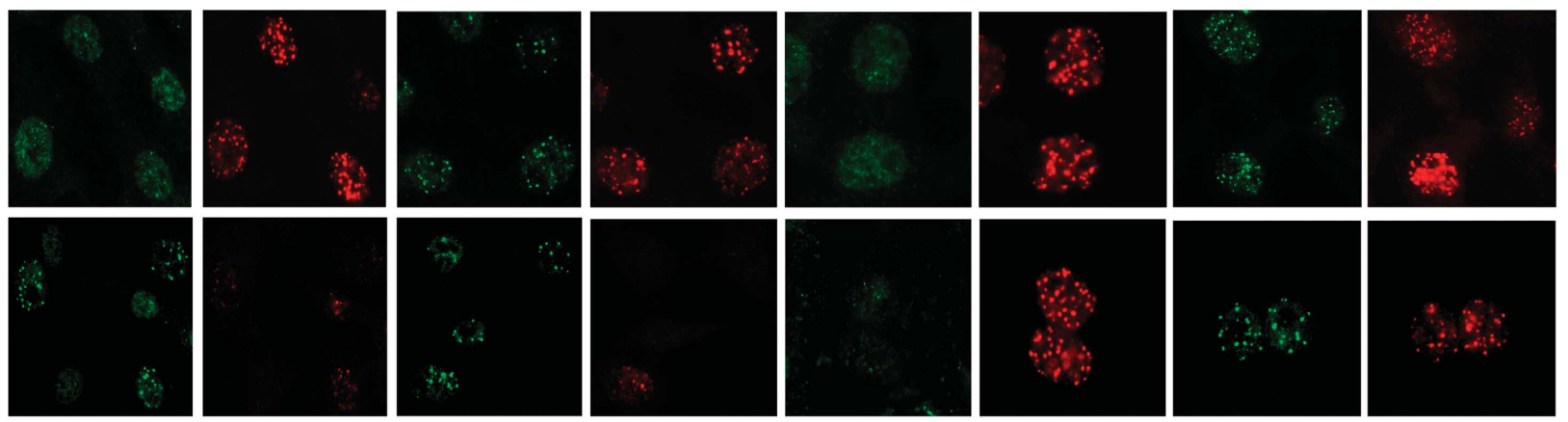

Figure 7 Downregulation of 53BP1 rescued Rad51 foci and HR in cells expressing MDC1K1840R. (A) MDC1 ${ }^{-/-}$MEFs reconstituted with FLAG-WT MDC1 or K1840R MDC1 were left untreated or treated with IR (2Gy). The formation of MDC1 and 53BP1 foci was then examined by immunofluorescence at indicated time points. (B) Quantification of the percentage of cells displaying MDC1 or 53BP1 foci. Each line represents three independent experiments. Error bars represent s.e.m. from three independent experiments. (C) MDC1 - / - MEFs reconstituted with WT or K1840R FLAG-MDC1 were treated with or without IR ( $2 \mathrm{~Gy}$ ). The formation of MDC1 and BRCA1 foci was then examined by immunofluorescence at indicated time points. Quantification of the percentage of cells displaying BRCA1 foci is shown. Error bars represent s.e.m. from three independent experiments. (D, E) MDC1 ${ }^{-/-}$MEFs reconstituted with WT or K1840R FLAG-MDC1 were infected with Ctrl or 53BP1 shRNA. Cells were treated with IR ( $2 \mathrm{~Gy}$ ), the formation of Rad51 and MDC1 foci was examined at indicated time points. Representative images are shown in (D), the quantification of the percentage of cells displaying Rad51 foci is shown in (E). Error bars represent s.e.m. from three independent experiments. (F) MEF cells as indicated were subjected to HR assay. Error bars represent s.e.m. from three independent experiments. ${ }^{* *} P<0.01$ two-tailed Student's $t$-test. 
buffer containing imidazole and eluted in SDS sample buffer with $200 \mathrm{mM}$ imidazole. Western blots were analyzed with indicated antibodies.

\section{In vivo ubiquitination assay}

Transfected HEK293T cells were treated with MG132 $\left(20 \mu \mathrm{g} \mathrm{ml}^{-1}\right)$ for $1 \mathrm{~h}$ followed by irradiation ( $5 \mathrm{~Gy}$ ). After $3 \mathrm{~h}$, cells were lysed in $120 \mu \mathrm{l}$ of $62.5 \mathrm{mM}$ Tris- $\mathrm{HCl}$ (pH 6.8), $2 \%$ SDS, $10 \%$ glycerol, $20 \mathrm{mM}$ $\mathrm{NEM}$ and $1 \mathrm{mM}$ iodoacetamide, boiled for $15 \mathrm{~min}$, diluted 10 times with NETN buffer containing protease inhibitors, $20 \mathrm{mM}$ NEM, and $1 \mathrm{mM}$ iodoacetamide, and centrifuged $\left(16000 \mathrm{~g}, 10 \mathrm{~min}, 4^{\circ} \mathrm{C}\right)$ to remove cell debris. The lysates were immunoprecipitated with EZview Red Anti-HA affinity gel (Sigma) or with anti- FLAG M2 affinity gel (Sigma) for $1.5 \mathrm{~h}$ at $4{ }^{\circ} \mathrm{C}$ with rocking and the precipitates were eluted in SDS sample buffer. Western blots were analyzed with indicated antibodies.

\section{In vitro sumoylation assay}

SUMO modification reactions were typically performed in a total volume of $20 \mu \mathrm{l}$ with $200 \mathrm{ng}$ of SUMO-activating enzyme (UBA2) (Boston Biochem), $100 \mathrm{ng}$ of Ubc9 (Boston Biochem), $1 \mu \mathrm{g}$ of SUMO1, SUMO2, or SUMO3 (Boston Biochem), $100 \mathrm{ng}$ of PIAS4 (Novus), and recombinant GST-MDC1 (aa1818-2089) bound to GSTsepharose $(20 \mu \mathrm{g})$. The $10 \times$ reaction buffer (Boston Biochem) was added with $4 \mathrm{mM}$ ATP-Mg. Reactions were incubated at $30^{\circ} \mathrm{C}$ for $1 \mathrm{~h}$ and stopped by addition of E1 stop buffer (Boston Biochem). The beads were washed with NETN and boiled in SDS sample buffer or subjected to in vitro ubiquitination assay.

\section{In vitro ubiquitination assay}

The Myc-RNF4 WT, RNF4 CS, or RNF4 $\Delta$ SIM were expressed in HEK $293 \mathrm{~T}$ and purified by immunoprecipitation with anti-Myc antibody. The Myc-RNF4 proteins were then eluted with Myc peptide (200ng $\mathrm{Hl}^{-1}$ ) (Sigma). The non-sumoylated or sumoylated GSTMDC1(aa1818-2089) bound to GST-sepharose were washed with Tris-HCl (50 mM, pH 7.5) and then incubated with $200 \mathrm{ng}$ of UbcH5a (Boston Biochem), 300 ng of ubiquitin-activating enzyme (UBE1) (Boston Biochem), $2 \mu \mathrm{g}$ of FLAG-ubiquitin (Boston Biochem), $3 \mu \mathrm{g}$ Myc-RNF4 in $40 \mu \mathrm{l}$ of reaction buffer ( $50 \mathrm{mM}$ Tris (pH 7.5), $2.5 \mathrm{mM}$ $\mathrm{MgCl}_{2}, 2 \mathrm{mM}$ ATP, and $2 \mathrm{mM}$ DTT). The mixture was incubated at $37^{\circ} \mathrm{C}$ for $40 \mathrm{~min}$, washed with NETN, and boiled in SDS sample buffer.

\section{HR assay}

A HeLa clone with the integrated HR reporter DR-GFP was a gift from Dr Maria Jasin (Sloan Kettering). One day after transfection with control or MDC1 siRNA, HeLa-DR-GFP cells were co-transfected with vector, siRNA-resistant WT HA-MDC1, or HA-MDC1 K1840R, and an I-SceI expression vector (pCBA-I-SceI). Cells were harvested 2 days after I-SceI transfection and subjected to flow cytometric analysis to examine recombination induced by I-Scel digestion. The parallel transfection with pEGFP-C1 was used to normalize for transfection efficiency. Results are presented as a percentage of control siRNA.

For HR assay with MEFs, the DR-GFP reporter was integrated into the genome of MDC1 $-/-$ MEFs by puromycin selection. Clones with a single copy of DR-GFP were identified by Southern blotting. These clones were then transfected with WT or K1840R FLAGMDC1, and selected with hygromycin. DR-GFP MEFs were transfected with I-SceI expression plasmid and subjected to flow cytometric analysis to examine recombination induced by I-SceI digestion. The parallel transfection with pEGFP-C1 was used to normalize for transfection efficiency.

\section{NHEJ assay}

The in vivo end-joining reporter plasmid pEGFP-Pem1-Ad2 has been described (Fattah et al, 2010). Prior to transfection, the pEGFP-Pem1Ad2 plasmid was digested with HindIII (NEB) for $12 \mathrm{~h}$. A pCherry plasmid (Clontech) was co-transfected with linearized pEGFP-Pem1Ad2 as a control for transfection efficiency. The cell line under analysis was subcultured a day before transfection and was 60 to $70 \%$ confluent for transfection. Transfections were performed using Lipofectamine 2000 (Invitrogen) according to manufacturer's instructions. Green (EGFP) and red (Cherry) fluorescence was measured by fluorescence-activated flow cytometry (FACS) $24 \mathrm{~h}$ later. For FACS analysis, cells were harvested, washed in $1 \times$ PBS, and fixed using $2 \%$ paraformaldehyde. FACS analysis was performed on a FACSCalibur instrument (BD Biosciences).

\section{Statistical analysis}

Student's $t$-test was performed. Error bars represent s.e.m. of three independent experiments.

\section{Supplementary data}

Supplementary data are available at The EMBO Journal Online (http://www.embojournal.org).

\section{Acknowledgements}

We thank Dr Richard Baer (Columbia University) for providing anti-CtIP antibody, Dr Junjie Chen (MD Anderson) for providing anti-53BP1 antibody, and $\mathrm{Dr}$ Xiaochun $\mathrm{Yu}$ for providing antiBRCA1 antibody. We thank Karl J Clark (Mayo Clinic) for providing the pKTol2c-linker plasmid. We also thank Dr Maria Jasin for providing DR-GFP reporter and Dr Eric A Hendrickson for providing pEGFP-Pem1-Ad2 plasmid. This work was supported by NIH (CA130996 and CA151329, ZL), National Natural Science Foundation of China (81102011 and 81101514, KL and JY), and Program for New Century Excellent Talents in University (NCET11-0388, JY)

Author contributions: KL, HZ and JY performed all the experiments described in the manuscript. KL, JY, LW, and ZL conceived the study and wrote the paper. ZL and JY supervised the project. All authors discussed and commented on the manuscript.

\section{Conflict of interest}

The authors declare that they have no conflict of interest.

\section{References}

Bekker-Jensen S, Rendtlew DJ, Fugger K, Gromova I, Nerstedt A, Lukas C, Bartek J, Lukas J, Mailand N (2010) HERC2 coordinates ubiquitin-dependent assembly of DNA repair factors on damaged chromosomes. Nat Cell Biol 12: 80-86; sup pp 81-112

Bouwman P, Aly A, Escandell JM, Pieterse M, Bartkova J, van der Gulden H, Hiddingh S, Thanasoula M, Kulkarni A, Yang Q, Haffty BG, Tommiska J, Blomqvist C, Drapkin R, Adams DJ, Nevanlinna H, Bartek J, Tarsounas M, Ganesan S, Jonkers J (2010) 53BP1 loss rescues BRCA1 deficiency and is associated with triple-negative and BRCA-mutated breast cancers. Nat Struct Mol Biol 17: 688-695

Bunting SF, Callen E, Wong N, Chen HT, Polato F, Gunn A, Bothmer A, Feldhahn N, Fernandez-Capetillo O, Cao L, Xu X, Deng CX, Finkel T, Nussenzweig M, Stark JM, Nussenzweig A (2010) 53BP1 inhibits homologous recombination in Brcal-deficient cells by blocking resection of DNA breaks. Cell 141: 243-254

Burgess RC, Rahman S, Lisby M, Rothstein R, Zhao X (2007) The Slx5-Slx8 complex affects sumoylation of DNA repair proteins and negatively regulates recombination. Mol Cell Biol 27: 6153-6162

Constantinou A, Chen XB, McGowan CH, West SC (2002) Holliday junction resolution in human cells: two junction endonucleases with distinct substrate specificities. EMBO J 21: 5577-5585

Dou H, Huang C, Singh M, Carpenter PB, Yeh ET (2010) Regulation of DNA repair through deSUMOylation and SUMOylation of replication protein A complex. Mol Cell 39: 333-345

Eladad S, Ye TZ, Hu P, Leversha M, Beresten S, Matunis MJ, Ellis NA (2005) Intra-nuclear trafficking of the BLM helicase to DNA damage-induced foci is regulated by SUMO modification. Hum Mol Genet 14: 1351-1365

Falck J, Coates J, Jackson SP (2005) Conserved modes of recruitment of ATM, ATR and DNA-PKcs to sites of DNA damage. Nature 434: $605-611$ 
Fattah F, Lee EH, Weisensel N, Wang Y, Lichter N, Hendrickson EA (2010) $\mathrm{Ku}$ regulates the non-homologous end joining pathway choice of DNA double-strand break repair in human somatic cells. PLoS Genet 6: e1000855

Galanty Y, Belotserkovskaya R, Coates J, Polo S, Miller KM, Jackson SP (2009) Mammalian SUMO E3-ligases PIAS1 and PIAS4 promote responses to DNA double-strand breaks. Nature 462: 935-939

Goldberg M, Stucki M, Falck J, D’Amours D, Rahman D, Pappin D, Bartek J, Jackson SP (2003) MDC1 is required for the intra-Sphase DNA damage checkpoint. Nature 421: 952-956

Harper JW, Elledge SJ (2007) The DNA damage response: ten years after. Mol Cell 28: 739-745

Helmink BA, Tubbs AT, Dorsett Y, Bednarski JJ, Walker LM, Feng Z, Sharma GG, McKinnon PJ, Zhang J, Bassing CH, Sleckman BP (2011) H2AX prevents CtIP-mediated DNA end resection and aberrant repair in G1-phase lymphocytes. Nature 469: 245-249

Ip SC, Rass U, Blanco MG, Flynn HR, Skehel JM, West SC (2008) Identification of Holliday junction resolvases from humans and yeast. Nature 456: 357-361

Jungmichel S, Stucki M (2010) MDC1: The art of keeping things in focus. Chromosoma 119: 337-349

Kosoy A, Calonge TM, Outwin EA, O'Connell MJ (2007) Fission yeast Rnf4 homologs are required for DNA repair. $J$ Biol Chem 282: 20388-20394

Lallemand-Breitenbach V, Jeanne M, Benhenda S, Nasr R, Lei M, Peres L, Zhou J, Zhu J, Raught B, de The H (2008) Arsenic degrades PML or PML-RARalpha through a SUMO-triggered RNF4/ubiquitin-mediated pathway. Nat Cell Biol 10: 547-555

Lee JH, Paull TT (2005) ATM activation by DNA double-strand breaks through the Mre11-Rad50-Nbs1 complex. Science 308: 551-554

Lieber MR, Ma Y, Pannicke U, Schwarz K (2003) Mechanism and regulation of human non-homologous DNA end-joining. Nat Rev Mol Cell Biol 4: 712-720

Lieber MR, Ma Y, Pannicke U, Schwarz K (2004) The mechanism of vertebrate nonhomologous DNA end joining and its role in $\mathrm{V}(\mathrm{D}) \mathrm{J}$ recombination. DNA Repair 3: 817-826

Lou Z, Chini CC, Minter-Dykhouse K, Chen J (2003) Mediator of DNA damage checkpoint protein 1 regulates BRCA1 localization and phosphorylation in DNA damage checkpoint control. J Biol Chem 278: 13599-13602

Lou Z, Minter-Dykhouse K, Franco S, Gostissa M, Rivera MA, Celeste A, Manis JP, van Deursen J, Nussenzweig A, Paull TT, Alt FW, Chen J (2006) MDC1 maintains genomic stability by participating in the amplification of ATM-dependent DNA damage signals. Mol Cell 21: 187-200

Mimitou EP, Symington LS (2009) Nucleases and helicases take center stage in homologous recombination. Trends Biochem Sci 34: $264-272$

Morris JR, Boutell C, Keppler M, Densham R, Weekes D, Alamshah A, Butler L, Galanty Y, Pangon L, Kiuchi T, Ng T, Solomon E (2009) The SUMO modification pathway is involved in the BRCA1 response to genotoxic stress. Nature 462: $886-890$

Moynahan ME, Pierce AJ, Jasin M (2001) BRCA2 is required for homology-directed repair of chromosomal breaks. Mol Cell 7: 263-272

Nimonkar AV, Genschel J, Kinoshita E, Polaczek P, Campbell JL, Wyman C, Modrich P, Kowalczykowski SC (2011) BLM-DNA2RPA-MRN and EXO1-BLM-RPA-MRN constitute two DNA end resection machineries for human DNA break repair. Genes Dev 25: $350-362$

Paull TT, Gellert M (1998) The 3' to 5' exonuclease activity of Mre 11 facilitates repair of DNA double-strand breaks. Mol Cell 1: 969979

Perry JJ, Tainer JA, Boddy MN (2008) A SIM-ultaneous role for SUMO and ubiquitin. Trends Biochem Sci 33: 201-208

Polo SE, Jackson SP (2011) Dynamics of DNA damage response proteins at DNA breaks: a focus on protein modifications. Genes Dev 25: 409-433
Prudden J, Pebernard S, Raffa G, Slavin DA, Perry JJ, Tainer JA, McGowan CH, Boddy MN (2007) SUMO-targeted ubiquitin ligases in genome stability. EMBO J 26: 4089-4101

Rogakou EP, Boon C, Redon C, Bonner WM (1999) Megabase chromatin domains involved in DNA double-strand breaks in vivo. J Cell Biol 146: 905-916

San Filippo J, Sung P, Klein H (2008) Mechanism of eukaryotic homologous recombination. Annu Rev Biochem 77: 229-257

Sartori AA, Lukas C, Coates J, Mistrik M, Fu S, Bartek J, Baer R, Lukas J, Jackson SP (2007) Human CtIP promotes DNA end resection. Nature 450: $509-514$

Shi W, Ma Z, Willers H, Akhtar K, Scott SP, Zhang J, Powell S (2008) Disassembly of MDC1 foci is controlled by ubiquitin-proteasomedependent degradation. J Biol Chem 283: 31608-31616

Stewart GS, Wang B, Bignell CR, Taylor AM, Elledge SJ (2003) $\mathrm{MDC} 1$ is a mediator of the mammalian DNA damage checkpoint. Nature 421: 961-966

Stucki M, Clapperton JA, Mohammad D, Yaffe MB, Smerdon SJ, Jackson SP (2005) MDC1 directly binds phosphorylated histone $\mathrm{H} 2 \mathrm{AX}$ to regulate cellular responses to DNA double-strand breaks. Cell 123: 1213-1226

Stucki M, Jackson SP (2004) MDC1/NFBD1: a key regulator of the DNA damage response in higher eukaryotes. DNA Repair 3: 953-957

Sun H, Leverson JD, Hunter T (2007) Conserved function of RNF4 family proteins in eukaryotes: targeting a ubiquitin ligase to SUMOylated proteins. EMBO J 26: 4102-4112

Sy SM, Chen J, Huen MS (2010) The 53BP1-EXPAND1 connection in chromatin structure regulation. Nucleus 1: 472-474

Takeda S, Nakamura K, Taniguchi Y, Paull TT (2007) Ctp1/CtIP and the MRN complex collaborate in the initial steps of homologous recombination. Mol Cell 28: 351-352

Tatham MH, Geoffroy MC, Shen L, Plechanovova A, Hattersley N, Jaffray EG, Palvimo JJ, Hay RT (2008a) RNF4 is a poly-SUMOspecific E3 ubiquitin ligase required for arsenic-induced PML degradation. Nat Cell Biol 10: 538-546

Tatham MH, Geoffroy MC, Shen L, Plechanovova A, Hattersley N, Jaffray EG, Palvimo JJ, Hay RT (2008b) RNF4 is a poly-SUMOspecific E3 ubiquitin ligase required for arsenic-induced PML degradation. Nat Cell Biol 10: 538-546

Thompson LH, Schild D (2002) Recombinational DNA repair and human disease. Mutat Res 509: 49-78

Uziel T, Lerenthal Y, Moyal L, Andegeko Y, Mittelman L, Shiloh Y (2003) Requirement of the MRN complex for ATM activation by DNA damage. EMBO J 22: 5612-5621

Warmerdam DO, Kanaar R (2010) Dealing with DNA damage: relationships between checkpoint and repair pathways. Mutat Res 704: 2-11

West SC (2003) Molecular views of recombination proteins and their control. Nat Rev Mol Cell Biol 4: 435-445

West SC (2009) The search for a human Holliday junction resolvase. Biochem Soc Trans 37: 519-526

Xie A, Hartlerode A, Stucki M, Odate S, Puget N, Kwok A, Nagaraju G, Yan C, Alt FW, Chen J, Jackson SP, Scully R (2007) Distinct roles of chromatin-associated proteins MDC1 and 53BP1 in mammalian double-strand break repair. Mol Cell 28: 1045-1057

Yurchenko V, Xue Z, Sadofsky MJ (2006) SUMO modification of human XRCC4 regulates its localization and function in DNA double-strand break repair. Mol Cell Biol 26: 1786-1794

Zhang C, Roberts TM, Yang J, Desai R, Brown GW (2006) Suppression of genomic instability by SLX5 and SLX8 in Saccharomyces cerevisiae. DNA Repair 5: 336-346

Zhang J, Ma Z, Treszezamsky A, Powell SN (2005) MDC1 interacts with Rad51 and facilitates homologous recombination. Nat Struct Mol Biol 12: 902-909

Zhou BB, Elledge SJ (2000) The DNA damage response: putting checkpoints in perspective. Nature 408: 433-439

Zhu Z, Chung WH, Shim EY, Lee SE, Ira G (2008) Sgs1 helicase and two nucleases Dna2 and Exo1 resect DNA double-strand break ends. Cell 134: 981-994 